\title{
Effect of Polymorphism and Application of Kinetic Models for the Evaluation of In Vitro Dissolution Profiles of an Eletriptan Hydrobromide Formulation
}

\author{
Pavan Kommavarapu ${ }^{1}{ }^{,}$, Arthanareeswari Maruthapillai ${ }^{1}$, Kamaraj Palanisamy ${ }^{1}$, and Ravi Teja Koya ${ }^{2}$ \\ ${ }^{1}$ Department of Chemistry, SRM University, Kattankulathur-603203, Tamilnadu, India \\ ${ }^{2}$ Department of Pharmaceutical analysis, Acharya Nagarjuna University-522510, Guntur, India \\ e-mail: kommavarapu.pavan@gmail.com
}

\begin{abstract}
The intent of this work was to study the effect of polymorphism on dissolution properties and to apply several methods to evaluate the dissolution profiles of immediate-release tablets containing alpha $(\alpha)$ and beta $(\beta)$ forms of eletriptan hydrobromide (EH). The polymorphs were characterized by X-ray diffraction (XRD), differential scanning calorimetry (DSC), and diffuse reflectance infrared Fourier transform spectroscopy (DRIFTS). The dissolution conditions were USP Apparatus 2 (paddle) with $900 \mathrm{~mL}$ of $0.1 \mathrm{~N} \mathrm{HCl}$ medium at a rotation speed of $100 \mathrm{rpm}$. The dissolution profiles were compared using model-independent, model-dependent, and statistical methods. In the model-independent approaches, dissolution efficiency $(D E)$, mean dissolution time $(M D T)$, difference factor $\left(f_{1}\right)$, and similarity factor $\left(f_{2}\right)$ were evaluated. A statistical assessment of $D E$ data was performed using ANOVA and the $t$-test. Dissolution kinetics was determined using model-dependent approaches in which nine drug release mathematical models were evaluated. The dissolution profile of the $\alpha$ formulation was best represented by the Weibull model, and the $\beta$ formulation by the Korsmeyer-Peppas model. The calculated fit factors infer that the two tablet formulations are similar with minor differences. Statistical and model-dependent approaches demonstrate that there may be a difference in the drug release mechanism.
\end{abstract}

KEYWORDS: Dissolution; eletriptan hydrobromide; model-dependent methods; model-independent methods; polymorphs.

\section{INTRODUCTION}

I letriptan hydrobromide is a triptan drug intended for the treatment of pain in nerve endings and associated symptoms caused by migraine headaches. It is a selective 5-hydroxytryptamine $1 \mathrm{~B} / 1 \mathrm{D}\left(5-\mathrm{HT}_{1 \mathrm{~B} / 1 \mathrm{D}}\right)$ receptor agonist that acts at serotonin $5-\mathrm{HT}_{1 \mathrm{~B}}$ receptors on intracranial blood vessels and $5-\mathrm{HT}_{1 \mathrm{D}}$ receptors on sensory nerve endings to reduce swelling of the blood vessels surrounding the brain thereby relieving the pain of a migraine attack (1-4).

$\mathrm{EH}$ is known to exist in both $\alpha$ and $\beta$ forms, and crystalline forms of each were developed to study their structural properties (5). EH drug product is formulated as immediate-release tablets. Earlier studies (6-10) have illustrated the possible effects of polymorphism on the bioavailability of some drugs. In the present study, the dissolution profiles of EH polymorph formulations were characterized to understand the potential differences between the two drug products, because various factors like manufacturing process and formulation can influence the therapeutic effect of a drug. *Corresponding author.

Dissolution Technologies | NOVEMBER 2015
In vitro dissolution has been accepted as an important tool in drug development. It can be used as a replacement for the evaluation of bioequivalence. Dissolution testing is used to determine long-term stability and drug product shelf life. Dissolution results can be used as a quality control tool for predicting in vivo performance of a drug product (11-13). Several guidelines on dissolution testing of solid dosage forms have been introduced by regulatory authorities like FDA. Dissolution testing can be used to estimate the effect of certain changes in the formulation and manufacturing processes; it provides a means to categorize between interbatch discrepancies and provides a specification for in vitro drug release allowing determination of in vivo suitability of the formulation (14). There are several kinetic models and theories that describe the drug dissolution profile relating the amount of drug dissolved from a pharmaceutical dosage system as a function of time. The transformation of the value obtained from the dissolution test is facilitated by a generic equation that mathematically deciphers the dissolution curve as a function of parameters related to dosage forms (15). 
A comparison of the dissolution profiles of two drug products can be done using model-independent methods, model-dependent (curve-fitting) methods, and statistical analysis. Model-independent approaches directly compare the dissolution data without having to depend on model functions. Mathematical models used to represent dissolution profiles require a suitable mathematical function that is either linear or nonlinear. After the model is selected, the dissolution profiles are compared and evaluated in terms of the model parameters, which provides an insight into the drug release mechanism $(15,16)$. The statistical comparison of the dissolution profiles can be performed by a $t$-test for the estimated parameters of the regression functions.

\section{Model-Independent Methods}

Model-independent approaches generate a single value from a dissolution profile, which provides a direct comparison of the dissolution data. Model-independent approaches include ratio tests and fit factors. The ratio test is performed by comparing the two mean dissolution times $(M D T)$, which are calculated by the formula:

$$
M D T=\frac{\sum_{i}^{n} \bar{t}_{i} \Delta M_{i}}{\sum_{i}^{n} \Delta M_{i}}
$$

where $i$ is the sample number, $n$ is the number of dissolution sample times, $\bar{t}=\left(t_{\mathrm{i} 1}+t_{\mathrm{i}}\right) / 2$ is the time at the midpoint between $t_{\mathrm{i}-1}$ and $t_{\mathrm{i}}$, and $\Delta M_{i}$ is the additional amount of drug dissolved between $t_{i-1}$ and $t_{i}$.

The primary constraint for comparison of two products or formulations or dosage forms is the in vitro dissolution profile. For the comparison of in vitro dissolution profiles, similarity and difference factors are recommended by the FDA. The similarity factor $\left(f_{2}\right)$ compares the closeness of two formulations (16). It can be calculated using the formula

$$
f_{2}=50 \cdot \log \left\{\left[1+\frac{1}{n} \sum_{t=1}^{n}\left(R_{t}-T_{t}\right)^{2}\right]^{-0.5} \times 100\right\}
$$

where $n$ is the number of dissolution sample times and $R_{t}$ and $T_{t}$ are the individual or mean percentage dissolved at each time point $t$ for the reference and test dissolution profiles, respectively. The $f_{1}$ factor focuses on the difference in percentage dissolved between reference and test at various time intervals. It can be mathematically computed using

$$
f_{1}=\left\{\left[\sum_{t=1}^{n}\left|R_{t}-T_{t}\right|\right] /\left[\sum_{t=1}^{n} R_{t}\right]\right\} \times 100
$$

The dissolution efficiency $(D E)$ of a pharmaceutical dosage form is defined as the area under the dissolution curve up to a certain time $t$ expressed as a percentage of the area of the rectangle described by $100 \%$ dissolution in the same time (17). It is calculated by the following equation:

$$
D E=\frac{\int_{t_{1}}^{t_{2}} y \cdot d t}{y_{100} \cdot\left(t_{2}-t_{1}\right)} \times 100
$$

where $y$ is the percentage drug dissolved at time $t$.

\section{Model-Dependent Methods}

Different mathematical models have been proposed to analyze dissolution profiles to determine the mechanism of drug release. The mathematical models of a dissolution profile can be deduced by a theoretical analysis of the process, but in most cases, because of the complexity of dosage forms, a theoretical foundation does not exist. As a result, semi-empirical or empirical models have to be used to fit dissolution data (15-18). The most common mathematical models used for the evaluation of dissolution profile are listed in Table 1.

Table 1. Kinetic Models for Dissolution Profile of Solid Dosage Forms

\begin{tabular}{|c|c|}
\hline Model & $\begin{array}{c}\text { Mathematical } \\
\text { Equation }\end{array}$ \\
\hline Zero-order kinetics & $Q_{t}=Q_{0}+K_{0} t$ \\
\hline First-order kinetics & $\ln Q_{t}=\ln Q_{0}+K_{1} t$ \\
\hline Second-order kinetics & $1 / Q_{t}=K t+1 / Q_{0}$ \\
\hline Third-order kinetics & $1 / Q_{t}{ }^{2}=K t+1 / Q_{0}{ }^{2}$ \\
\hline Korsmeyer-Peppas & $Q_{t}=k t^{n}$ \\
\hline Weibull & $\log \left[-\ln \left(1-Q_{t} / Q_{\infty}\right)\right]=\mathrm{b} \times \log t-\log a$ \\
\hline Hixson-Crowell & $Q_{0}{ }^{1 / 3}-Q_{t}{ }^{1 / 3}=K_{S} t$ \\
\hline Higuchi & $Q_{t}=K_{\mathrm{H}} v t$ \\
\hline Baker-Lonsdale & $(3 / 2)\left[1-\left(1-Q_{t} / Q_{\infty}\right)^{2 / 3}\right]\left(Q_{t} / Q_{\infty}\right)=K_{t}$ \\
\hline
\end{tabular}

The main objective of this work was to establish the kinetics of EH polymorphs in their respective solid dosage forms and to compare their dissolution profiles using model-dependent and model-independent approaches.

\section{MATERIALS AND METHODS}

\section{Chemicals and Materials}

EH polymorph final dosage forms were obtained from PharmaTrain (Hyderabad, India). Hydrochloric acid was of Guaranteed Reagent (GR) grade. Microcrystalline cellulose (FMC biopolymer), lactose anhydrous (DMV), sodium croscarmellose (FMC biopolymer), and magnesium stearate (Avantor) were used. Ultra pure water (Millipore, USA) was used for the dissolution medium and throughout the analysis. 


\section{Formulation of Eletriptan Tablets}

Eletriptan API and all excipients (Table 2) were accurately weighed. The powder was blended in a poly bag by tumbling for five minutes. The blend was transferred directly into the hopper of a single-punch tablet machine (Erweka, GmbH, Germany) having a caplet-shaped, concave punch and was compressed manually at room temperature.

Table 2. Formulation of Eletriptan Tablets

\begin{tabular}{|c|c|c|c|c|}
\hline \multirow{2}{*}{ Ingredient } & \multicolumn{2}{|c|}{$\begin{array}{c}\text { Weight/Tablet (mg) } \\
\text { Formulation }\end{array}$} & \multicolumn{2}{c|}{$\begin{array}{c}\text { Percentage (w/w) } \\
\text { Formulation }\end{array}$} \\
\cline { 2 - 5 } & Form. 1 & Form. 2 & Form. 1 & Form. 2 \\
\hline Eletriptan $\alpha$ form & 50.0 & - & 25.0 & - \\
\hline Eletriptan 6 form & - & 50.0 & - & 25.0 \\
\hline $\begin{array}{c}\text { Microcrystalline } \\
\text { cellulose }\end{array}$ & 72.5 & 72.5 & 36.25 & 36.25 \\
\hline $\begin{array}{c}\text { Lactose anhydrous } \\
\text { impalpable }\end{array}$ & 71.0 & 71.0 & 35.5 & 35.5 \\
\hline Sodium croscarmellose & 4.0 & 4.0 & 2.0 & 2.0 \\
\hline Magnesium stearate & 2.5 & 2.5 & 1.25 & 1.25 \\
\hline
\end{tabular}

\section{Characterization of Eletriptan Formulation}

\section{$X$-ray Diffractometry}

$X$-ray diffraction (XRD) patterns were collected using a Bruker D8 ADVANCE X-ray diffractometer with a Cu anode and LYNXEYE XE detector. EH $\alpha$ and $B$ forms were scanned from $3^{\circ} 2 \vartheta$ to $45^{\circ} 2 \vartheta$, with step size of $0.01^{\circ} 2 \vartheta$ and time per step of $0.1 \mathrm{sec}$ for the purpose of identification. The instrument was operated at $40 \mathrm{kV}$ and a current of 40 $\mathrm{mA}$. $\mathrm{V}_{20 \mathrm{~mm}}$ variable divergence and anti-scattering slits were used, and a Ni filter was used in the secondary beam path. DIFFRAC.EVA software was used for data processing and evaluation.

\section{Differential Scanning Calorimetry}

Differential scanning calorimetry (DSC) of $\mathrm{EH} \alpha$ and $b$ forms was performed using a Mettler Toldeo Model DSC 1 STAR System. Samples were accurately weighed in aluminum pans and sealed. Thermograms were obtained from 25 to $300^{\circ} \mathrm{C}$ at a heating rate of $10^{\circ} \mathrm{C} / \mathrm{min}$. Nitrogen was used as the purge gas at a flow rate of $50 \mathrm{~mL} / \mathrm{min}$. An aluminum pan was used as a reference.

\section{Diffuse Reflectance Infrared Fourier} Transform Spectroscopy

Spectra of the drug powder were recorded using diffuse reflectance infrared Fourier transform spectroscopy (DRIFTS) at room temperature in the range of 4000$400 \mathrm{~cm}^{-1}$ (Perkin Elmer Spectrum 100) with a diffuse reflectance sampling accessory. Sample mixtures were well ground to ensure homogeneity and increase relative reflectance, then placed in small sample cups and kept on the sample holder. The spectra were recorded with a resolution of $4.0 \mathrm{~cm}^{-1}$. Sixteen scans were collected for each spectrum. A background spectrum of ground $\mathrm{KBr}$ powder was obtained for each experimental condition. Spectroscopic manipulation such as smoothing and normalization was performed using software.

\section{In Vitro Study}

\section{Dissolution Test Conditions}

The dissolution test was performed using a Distek Evolution dissolution test system, model $6300(n=12)$. Dissolution was performed using USP Apparatus 2 (paddle) in $900 \mathrm{~mL}$ of medium at $37 \pm 0.5^{\circ} \mathrm{C}$ at a rotation speed of $100 \mathrm{rpm}$. Sample aliquots were withdrawn at $5,10,15,30$, and $45 \mathrm{~min}$ and replaced with equal volumes of fresh medium to maintain a constant total volume. All dissolution samples were analyzed by UV-vis spectrophotometry at $272 \mathrm{~nm}$ versus dissolution medium as a blank using a $10-\mathrm{mm}$ cell.

The dissolution medium was $0.1 \mathrm{~N}$ hydrochloric acid. Hydrochloric acid $(85 \mathrm{~mL}$ ) was transferred to a 10 -L flask containing $5000 \mathrm{~mL}$ of water, mixed, brought to volume with water, and mixed well. The prepared dissolution medium was degassed before use.

To prepare a stock standard solution, $53.5 \mathrm{mg}$ of EH API equivalent to $44.2 \mathrm{mg}$ of eletriptan was weighed into a $100-\mathrm{mL}$ volumetric flask, dissolved in $50 \mathrm{~mL}$ of dissolution medium by sonication, diluted to volume with dissolution medium, and mixed well.

To prepare the standard solution, $5 \mathrm{~mL}$ of standard stock solution was diluted to $50 \mathrm{~mL}$ with dissolution medium and mixed well.

Six dissolution vessels were filled with $900 \mathrm{~mL}$ of medium and equilibrated at $37 \pm 0.5^{\circ} \mathrm{C}$. One 40 - $\mathrm{mg}$ strength tablet was placed in each basket, and the dissolution was started. After a specified time, $10 \mathrm{~mL}$ of sample was withdrawn from each dissolution vessel and filtered through a $0.45-\mu$ PVDF filter.

\section{Procedure}

After the first $3 \mathrm{~mL}$ of filtrate was discarded, the absorbance of the standard and test solutions was measured at $272 \mathrm{~nm}$ versus the dissolution medium as a blank in $10-\mathrm{mm}$ cells. The percentage drug dissolved was calculated using the formula

$\%$ Drug dissolved $=\frac{A_{\mathrm{T}}}{A_{\mathrm{S}}} \times \frac{W_{\mathrm{S}}}{100} \times \frac{5}{50} \times \frac{900}{1} \times \frac{P}{100} \times \frac{100}{L} \times \frac{382.52}{463.40}$ 
where $A_{T}$ is the absorbance of the test solution, $A_{S}$ is the absorbance the standard solution, $W_{S}$ is the weight of API in $\mathrm{mg}$ in the standard solution, $P$ is drug potency, $L$ is the label amount of drug per tablet in $\mathrm{mg}, 382.52$ is the molecular weight of eletriptan, and 463.40 is the molecular weight of EH.

\section{RESULTS AND DISCUSSIONS}

The diffraction patterns from the XRD study (Figure 1) indicate a difference between the crystalline nature of the $\alpha$ and $B$ forms of $\mathrm{EH}$, with characteristic peaks represented in Table 3. Drug excipient interactions and compatibility studies were performed using XRPD. Figure 2 represents the overlaid diffraction patterns of $\mathrm{EH} \alpha$ polymorph, EH $B$ polymorph, magnesium stearate, lactose anhydrous impalpable, microcrystalline cellulose, sodium croscarmellose, processed placebo, EH $\alpha$-polymorph formulation, and $\mathrm{EH}$ 6-polymorph formulation. There are no extra peaks in the diffraction pattern of the processed placebo other than the peaks from each excipient, which shows excipient-excipient interaction compatibility. In the diffraction pattern of the EH $\alpha$-polymorph formulation, there are no peak other than those of EH $\alpha$ polymorph and processed placebo. Similarly, the diffraction pattern of the EH B-polymorph formulation contains only peaks belonging to $\mathrm{EH} B$ polymorph and processed placebo. This study shows the physical stability and drug-excipient compatibility of the $\mathrm{EH} \alpha$-polymorph formulation and the EH 6-polymorph formulation.

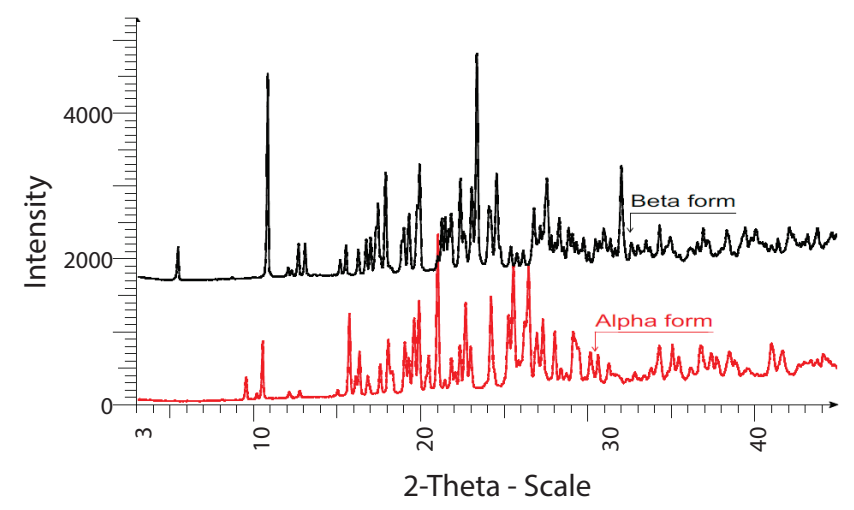

Figure 1. Diffractogram of $\alpha$ and $B$ forms of eletriptan hydrobromide.

The DSC thermogram (Figure 3) shows that the melting point range of eletriptan is $169-171{ }^{\circ} \mathrm{C}$ for the $\alpha$ form and $147-149{ }^{\circ} \mathrm{C}$ for the $\beta$ form. The two polymorphic mixtures of eletriptan were characterized by DRIFTS as shown in Figure 4. The polymorphs have distinct vibrational frequencies, which may serve for identification. The most prominent differences are in the region of $557.0 \mathrm{~cm}^{-1}, 769.2 \mathrm{~cm}^{-1}, 823.1 \mathrm{~cm}^{-1}, 870.2 \mathrm{~cm}^{-1}$, $1123.2 \mathrm{~cm}^{-1}$, and $1409.1 \mathrm{~cm}^{-1}$.

Table 3. Characteristic Peaks of Eletriptan Polymorphs Using X-ray Diffraction

\begin{tabular}{|c|c|c|c|}
\hline \multicolumn{2}{|c|}{ Eletriptan $\alpha$-form } & \multicolumn{2}{|c|}{ Eletriptan 8 -form } \\
\hline 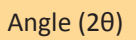 & Intensity \% & Angle $(2 \theta)$ & Intensity \% \\
\hline 9.491 & 15.7 & 5.408 & 15.6 \\
\hline 10.141 & 6.3 & 8.706 & 2.8 \\
\hline 10.485 & 37 & 10.789 & 91.3 \\
\hline 12.085 & 7.1 & 12.017 & 6.6 \\
\hline 12.715 & 7.6 & 12.232 & 5.4 \\
\hline 15.007 & 8.1 & 12.632 & 17.1 \\
\hline 15.695 & 53 & 13.04 & 17.2 \\
\hline 16.096 & 16.3 & 15.15 & 9.8 \\
\hline 16.303 & 30.7 & 15.487 & 16.3 \\
\hline 16.799 & 16.5 & 16.238 & 14.4 \\
\hline 17.551 & 23.4 & 16.706 & 18.9 \\
\hline 18.032 & 37.8 & 16.972 & 19.9 \\
\hline 18.262 & 36.6 & 17.407 & 34.6 \\
\hline 19.025 & 27.1 & 17.877 & 48.1 \\
\hline 19.252 & 50.4 & 18.96 & 24.1 \\
\hline 19.587 & 61.1 & 19.283 & 30 \\
\hline 19.88 & 28.5 & 19.754 & 33.1 \\
\hline 20.457 & 100 & 19.911 & 51.6 \\
\hline 21.005 & 14.1 & 21.023 & 11.7 \\
\hline 21.435 & 26.9 & 21.25 & 28.1 \\
\hline 21.825 & 18.5 & 21.457 & 28.6 \\
\hline 22.055 & 34.5 & 21.81 & 30.1 \\
\hline
\end{tabular}

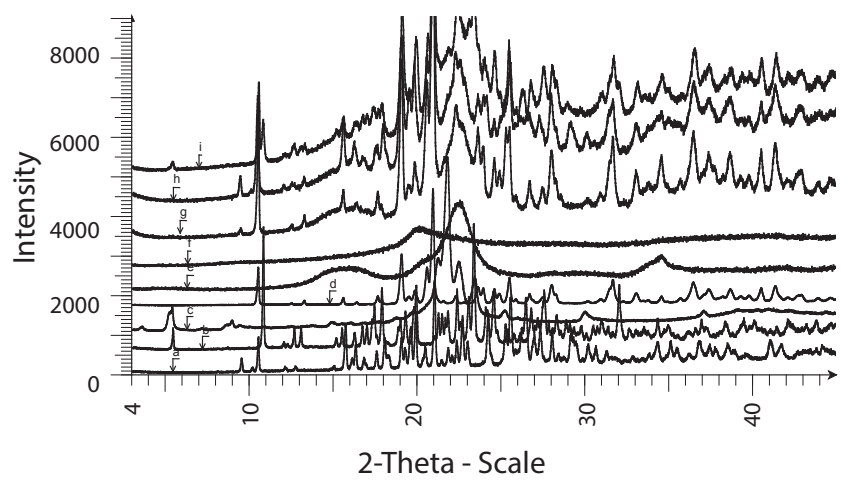

Figure 2. Overlay of X-ray powder diffraction patterns of (a) EH polymorph- $\alpha$, (b) EH polymorph-b, (c) magnesium stearate, (d) lactose anhydrous impalable, (e) microcrystalline cellulose, (f) sodium croscarmellose, (g) processed placebo, (h) EH a-polymorph formulation, and (i) EH 6-polymorph formulation. 


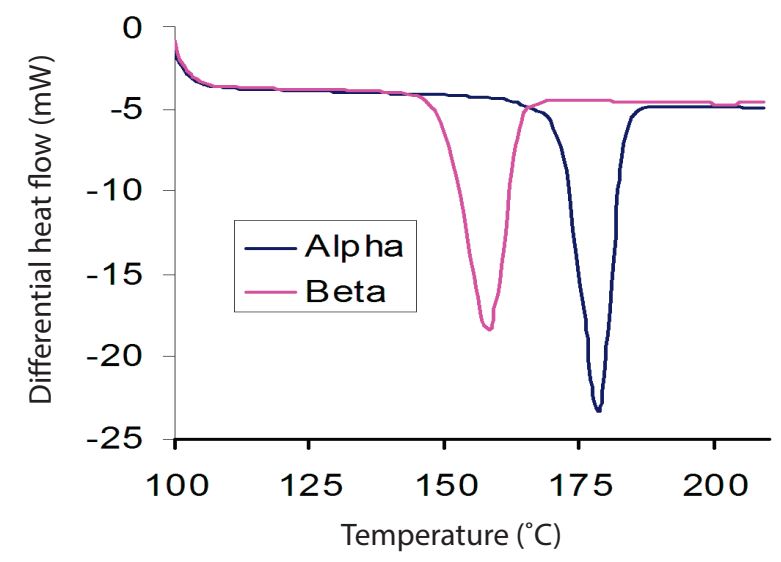

Figure 3. DSC thermogram of $\alpha$ and $B$ forms of eletriptan hydrobromide.

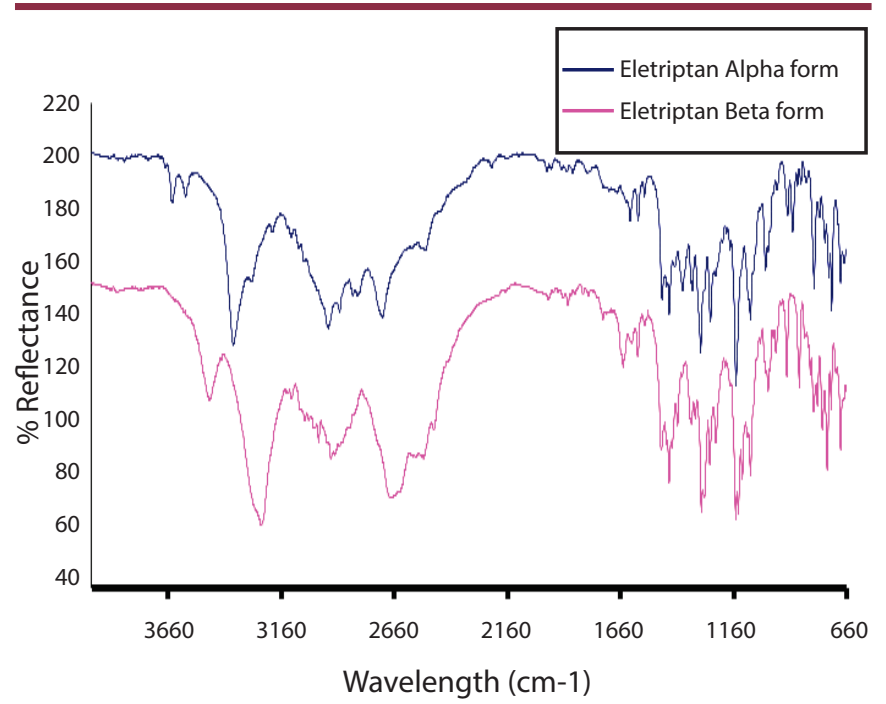

Figure 4. IR spectra of $\alpha$ and $B$ forms of eletriptan hydrobromide.

\section{Dissolution Apparatus and Paddle Rotation Speed Selection}

The apparatus and paddle rotation speed must provide proper drug product distribution in the dissolution medium and should maintain the discriminatory power of the dissolution method. USP Apparatus 2 was selected because it is standard for tablet formulations. To demonstrate method robustness, paddle speeds of $50 \pm$ $5 \mathrm{rpm}, 75 \pm 5 \mathrm{rpm}$, and $100 \pm 5 \mathrm{rpm}$ were assessed with samples collected at specified time points. The dissolution profiles of the $\alpha$ and $\beta$ formulations at different paddle rotation speeds are represented in Figure 5. These dissolution profiles show high variability in drug release for both formulations at $50 \pm 5 \mathrm{rpm}$ and $75 \pm 5 \mathrm{rpm}$, which reveals a lack of robustness. The lower variability of data for $100 \pm 5 \mathrm{rpm}$ demonstrates the method is robust at this speed.
Visual observation of tablet dissolution at $50 \pm 5 \mathrm{rpm}$ and $75 \pm 5 \mathrm{rpm}$ revealed the presence of coning after disintegration. This resulted in incomplete dissolution due to trapping of the drug in the cone of excipients at the bottom of the dissolution vessels. This phenomenon is associated with certain types of tablet formulations and with the poor hydrodynamics associated with Apparatus 2 at paddle speeds of 50 and $75 \mathrm{rpm}$ and resulted in high variability in dissolution data observed by varying $\pm 5 \mathrm{rpm}$.

When the paddle speed was increased to $100 \mathrm{rpm}$, the increased turbulence in the vessel prevented cone formation and exposed all tablet granules to the dissolution medium. Consequently, the dissolution profile more accurately reflected the dissolution of the tablet, not system hydrodynamics, and demonstrated a more rugged test procedure.

Results for the in vitro dissolution tests of EH polymorph tablets and statistical properties are given in Table 4. For both $\alpha$ and $\theta$ formulations, the drug dissolved completely in $30 \mathrm{~min}$. The dissolution profile of the $\alpha$ formulation
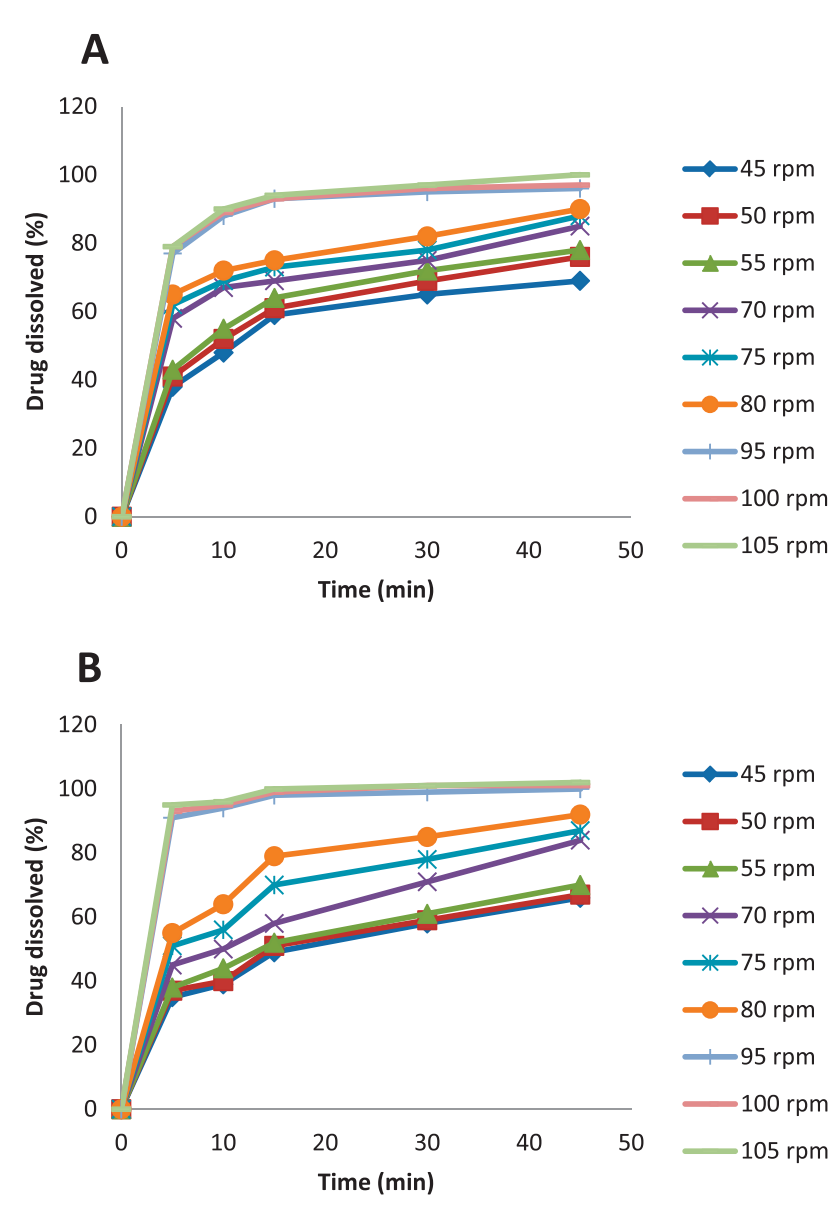

Figure 5. Dissolution profiles of (A) $\alpha$ and (B) $B$ formulations at $50 \pm 5 \mathrm{rpm}, 75 \pm 5 \mathrm{rpm}$, and $100 \pm 5 \mathrm{rpm}$. 
exhibits three stages of dissolution, and the dissolution profile of the $B$ formulation exhibits two stages of dissolution. A large amount of drug dissolved in the first 5 min for both formulations (i.e., approximately $80 \%$ for the $\alpha$ formulation and $90 \%$ for the 6 formulation), which is the initial step. In the second step for the $\alpha$ formulation, about $12 \%$ more drug was released in $10 \mathrm{~min}$, thus approximately $90 \%$ of drug was released in $10 \mathrm{~min}$. For the 6 formulation, only about $2 \%$ more drug was released in $10 \mathrm{~min}$ and about $6 \%$ more drug was released in 15 min (i.e., about $100 \%$ drug was released within $15 \mathrm{~min}$ ). About 8\% more drug was released for the $\alpha$ formulation between 10 and $30 \mathrm{~min}$.

Table 4. Dissolution Data for EH Polymorph Tablets

\begin{tabular}{|c|c|c|c|c|c|}
\hline $\begin{array}{c}\text { Time } \\
(\mathbf{m i n})\end{array}$ & Form & $\begin{array}{c}\text { \% Mean } \\
\text { Drug } \\
\text { dissolved }\end{array}$ & $\begin{array}{c}\text { Standard } \\
\text { deviation ( } \sigma)\end{array}$ & Minimum & Maximum \\
\hline \multirow{2}{*}{5} & $\alpha$ & 79 & 5.7 & 73 & 88 \\
\cline { 2 - 6 } & $\beta$ & 93 & 2.3 & 89 & 95 \\
\hline \multirow{2}{*}{10} & $\alpha$ & 89 & 3.4 & 86 & 94 \\
\cline { 2 - 6 } & $\beta$ & 95 & 2.1 & 92 & 97 \\
\hline \multirow{2}{*}{15} & $\alpha$ & 93 & 2.0 & 91 & 96 \\
\cline { 2 - 6 } & $\beta$ & 99 & 1.6 & 97 & 101 \\
\hline \multirow{2}{*}{30} & $\alpha$ & 96 & 0.4 & 96 & 97 \\
\cline { 2 - 6 } & $\beta$ & 101 & 1.3 & 99 & 102 \\
\hline \multirow{2}{*}{45} & $\alpha$ & 97 & 0.8 & 96 & 98 \\
\cline { 2 - 6 } & $\beta$ & 101 & 1.4 & 99 & 101 \\
\hline
\end{tabular}

\section{Model-Independent Methods}

The MDT values for the $\alpha$ and $B$ formulations of EH, shown in Table 5, were used to compare the dissolution profiles and to establish an in vitro-in vivo relationship. The MDT values show that both the $\alpha$ and $B$ formulations have similar dissolution profiles before $15 \mathrm{~min}$ and from 15 to $30 \mathrm{~min}$; these values indicate that there is a variation in the release mechanism. Since the MDT values are quite low for both formulations, the release rate is higher, which indicates lower drug-retarding ability of the polymer.

The $D E$ values for the $\alpha$ and $B$ formulations are shown in Table 5. $D E$ values are related to the actual amount of drug dissolved in the dissolution medium and thus lead to a better extrapolation for in vivo performance. From the analysis of variance (ANOVA-single factor) results of $D E$ data, the $F$ value (13.6) is greater than $F_{\text {crit }}(3.9)$ and the $p$ value (0.001) is extremely low; therefore, there is a significant difference between the groups. To assess the performance of the two formulations statistically, the $t$-test (two-sample assuming equal variances) was performed. The absolute value of $t$-stat $(0.5853)$ is less than $t$ critical value (2.30), and the $p$ value $(0.57)$ is greater than 0.05 , thus there is a statistical difference in the performances of the two formulations with respect to dissolution. From ANOVA single-factor analysis on the $M D T$ results, the $F$ value (5.08) is below $F_{\text {crit }}$ (5.99) and the $p$ value (0.07) is greater than 0.05 , so there is no significant difference between the groups. Application of the $t$-test (two-sample assuming equal variances) to the MDT results gave an absolute value of $t$-stat (2.25) that is less than the $t$-critical value (2.44); hence, there is a statistical difference in the performance of the two formulations with respect to MDT.

Table 5. MDT and DE Values for EH $\alpha$ and $B$ Formulations

\begin{tabular}{|c|c|c|c|c|}
\hline \multirow{2}{*}{ Time (min) } & \multicolumn{2}{|c|}{$M D T(\min )$} & \multicolumn{2}{c|}{$D E$} \\
\cline { 2 - 5 } & $\boldsymbol{\alpha}$ & $\boldsymbol{\beta}$ & $\boldsymbol{\alpha}$ & $\boldsymbol{\beta}$ \\
\hline 5 & - & - & 39.5 & 46.5 \\
\hline 10 & 3.06 & 2.61 & 61.7 & 70.2 \\
\hline 15 & 3.47 & 3.01 & 71.5 & 79.2 \\
\hline 30 & 4.06 & 3.20 & 83.0 & 89.3 \\
\hline 45 & 4.41 & 3.20 & 87.5 & 92.9 \\
\hline
\end{tabular}

Fit factors are quantitative methods used to compare different dissolution profiles. The similarity factor $\left(f_{2}\right)$ has been adopted by FDA as a criterion for the assessment of the similarity between two dissolution profiles. The difference factor $\left(f_{1}\right)$ measures the percent error between two curves over all time points (17). The $\alpha$ formulation was selected as the reference profile in this study, and the fit factor values for $\alpha$ and $B$ formulations of $\mathrm{EH}$ are presented in Table 6. A similarity factor value of 53 at $45 \mathrm{~min}$ indicates the sameness of the two products, and the difference factor value of 8 at 45 min shows minor differences between the two products.

Table 6. Fit Factors for $\alpha$ - and B-Formulations Based on the Average of Six Tablets

\begin{tabular}{|c|c|c|c|}
\hline \multirow{2}{*}{ Time $(\min )$} & \multicolumn{3}{|c|}{ Fit Factor } \\
\cline { 2 - 4 } & Form & $f_{2}$ & $f_{1}$ \\
\hline 5 & $\alpha / \beta$ & 58 & 18 \\
\hline 10 & $\alpha / \beta$ & 56 & 12 \\
\hline 15 & $\alpha / \beta$ & 54 & 10 \\
\hline 30 & $\alpha / \beta$ & 53 & 9 \\
\hline 45 & $\alpha / \beta$ & 53 & 8 \\
\hline
\end{tabular}




\section{Model-Dependent Methods}

The dissolution profiles were used to evaluate the kinetics of drug release. Nine different kinetic models were evaluated at 30 and $45 \mathrm{~min}$. Coefficient of determination $\left(R^{2}\right)$ values are shown in Table 7 for the different kinetic models used (Table 1). For the $\alpha$ formulation, the dissolution profiles are best described by the Weibull model. According to this model, at $30 \mathrm{~min}$ the $R 2$ value is $9.77 \times 10^{-1}$, and at $45 \mathrm{~min}, R^{2}=9.70 \times 10^{-1}$. Because this is an empirical model, it presents some deficiencies. For example, the model can describe only the dissolution drug release profile but not the release kinetics, and it is of limited use for establishing in vivo-in vitro correlations (19). The 6 formulation is best described by the Korsmeyer-Peppas model, which is a semi-empirical model relating drug release exponentially to elapsed time. The $R^{2}$ values at 30 and $45 \mathrm{~min}$ are $9.08 \times 10^{-1}$ and $8.73 \times 10^{-1}$, respectively. This model is used to analyze the release of pharmaceutical polymeric dosage forms when the release mechanism is not well known or when more than one type of release phenomenon could be involved.

Table 7. Mathematical Models and Coefficient of Determination $\left(R^{2}\right)$ for the Evaluation of Dissolution Profiles

\begin{tabular}{|c|c|c|c|c|}
\hline \multirow{2}{*}{ Model } & \multicolumn{2}{|c|}{$\boldsymbol{R}^{\mathbf{2}}$ for $\mathbf{3 0} \mathrm{min}$} & \multicolumn{2}{c|}{$\boldsymbol{R}^{\mathbf{2}}$ for $45 \mathrm{~min}$} \\
\cline { 2 - 5 } & $\boldsymbol{\alpha}$ & $\boldsymbol{\beta}$ & $\boldsymbol{\alpha}$ & $\boldsymbol{\beta}$ \\
\hline $\begin{array}{c}\text { Zero-order } \\
\text { kinetics }\end{array}$ & $7.28 \times 10^{-1}$ & $7.87 \times 10^{-1}$ & $6.71 \times 10^{-1}$ & $6.79 \times 10^{-1}$ \\
\hline $\begin{array}{c}\text { First-order } \\
\text { kinetics }\end{array}$ & $7.07 \times 10^{-1}$ & $7.84 \times 10^{-1}$ & $6.49 \times 10^{-1}$ & $6.75 \times 10^{-1}$ \\
\hline $\begin{array}{c}\text { Second-order } \\
\text { kinetics }\end{array}$ & $6.86 \times 10^{-1}$ & $7.81 \times 10^{-1}$ & $6.26 \times 10^{-1}$ & $6.72 \times 10^{-1}$ \\
\hline $\begin{array}{c}\text { Third-order } \\
\text { kinetics }\end{array}$ & $6.66 \times 10^{-1}$ & $7.77 \times 10^{-1}$ & $6.03 \times 10^{-1}$ & $6.68 \times 10^{-1}$ \\
\hline $\begin{array}{c}\text { Korsmeyer- } \\
\text { Peppas }\end{array}$ & $9.12 \times 10^{-1}$ & $9.08 \times 10^{-1}$ & $8.82 \times 10^{-1}$ & $8.73 \times 10^{-1}$ \\
\hline Weibull & $9.77 \times 10^{-1}$ & $7.96 \times 10^{-1}$ & $9.70 \times 10^{-1}$ & $8.70 \times 10^{-1}$ \\
\hline Hixson-Crowell & $7.14 \times 10^{-1}$ & $7.85 \times 10^{-1}$ & $6.56 \times 10^{-1}$ & $6.77 \times 10^{-1}$ \\
\hline Higuchi & -14.0 & -113.0 & -22.5 & -169.5 \\
\hline Baker-Lonsdale & $8.41 \times 10^{-1}$ & $9.01 \times 10^{-1}$ & $8.03 \times 10^{-1}$ & $7.90 \times 10^{-1}$ \\
\hline
\end{tabular}

Based on the values of $R^{2}$, root-mean-square error (RMSE), Akaike information criterion (AIC), and Bayesian information criterion (BIC), the best mathematical model describing dissolution profile was selected. RMSE, AIC, and BIC values are shown in Table 8. The RMSE, AIC, and BIC values for the $\alpha$ formulation following Weibull model and $\beta$ formulation following Korsmeyer-Peppas model are low when compared with the those of the other models, so these two models were selected to describe the dissolution profiles of the EH polymorphs.
Table 8. Model Selection Parameters for Kinetic Models

\begin{tabular}{|c|c|c|c|c|c|c|}
\hline \multirow{2}{*}{ Model } & \multicolumn{2}{|c|}{ RMSE } & \multicolumn{2}{c|}{ AIC } & \multicolumn{2}{c|}{ BIC } \\
\cline { 2 - 7 } & $\boldsymbol{\alpha}$ & $\boldsymbol{B}$ & $\boldsymbol{\alpha}$ & $\boldsymbol{B}$ & $\boldsymbol{\alpha}$ & $\boldsymbol{6}$ \\
\hline Zero-order & 3.34 & 1.32 & 19.20 & 11.76 & 17.97 & 10.54 \\
\hline First-order & 3.47 & 1.34 & 19.50 & 11.89 & 18.27 & 10.67 \\
\hline Second-order & 3.62 & 1.36 & 19.84 & 12.03 & 18.62 & 10.80 \\
\hline Third-order & 3.80 & 1.39 & 20.23 & 12.17 & 19.01 & 10.94 \\
\hline Korsmeyer-Peppas & 1.96 & 0.88 & 14.91 & 8.53 & 13.68 & 7.30 \\
\hline Weibull & 0.88 & 5.64 & 8.49 & 23.38 & 7.26 & 22.16 \\
\hline Hixson-Crowell & 3.43 & 1.33 & 19.40 & 11.85 & 18.17 & 10.62 \\
\hline Higuchi & 24.84 & 30.55 & 35.25 & 36.90 & 34.02 & 35.67 \\
\hline Baker-Lonsdale & 89.48 & 96.79 & 45.50 & 46.13 & 44.27 & 44.90 \\
\hline
\end{tabular}

\section{Application of the Present Study}

The model-dependent, model-independent, and statistical approaches used here for the comparison of dissolution profiles were applicable and useful. The application and assessment of model-dependent methods are more complicated, and these methods present an acceptable model approach to the true relationship between the dependent and independent variables. Statistical and model-dependent approaches have very narrow limits and are more discriminative than model-independent approaches. Use of statistical approaches for the comparison of dissolution profiles helps to contrast the data point-by-point and to determine the source of differences among the variables. The application and elucidation of model-independent methods are easier where only one value is obtained to describe the proximity of the two dissolution profiles. The application of fit factors is well suited to the qualitative determination of "similarity" as required by the FDA guidance (20). However, they do not provide information on individual batches, including their consistency. In contrast, $D E$ provides such information and is well matched for making quantitative comparisons. Using a combination of the above approaches, it is possible to obtain detailed information about dissolution data, which can be useful in formulation development and in understanding the different release mechanisms.

\section{CONCLUSIONS}

In vitro dissolution was carried out to evaluate the performance characteristics of $\mathrm{EH}$ polymorph tablets. Different model-independent and model-dependent approaches were used to compare the dissolution profiles. Based on fit factors using a difference and 
similarity approach, the two formulations appear similar with minor differences. Results from statistical and modeldependent approaches, which are complicated and more discriminative than model-independent methods, suggest that there is a difference between the performance of the two products and the release mechanisms.

\section{CONFLICT OF INTEREST}

No conflict of interest has been declared by the authors.

\section{REFERENCES}

1. Färkkilä, M.; Kallela, M. Eletriptan review. Expert Opin. Pharmacother. 2005, 6 (4), 625-630. DOI: 10.1517/14656566.6.4.625.

2. McCormack, P. L.; Keating, G. M. Eletriptan: A Review of its Use in the Acute Treatment of Migraine. Drugs 2006, 66 (8), 1129-1149. DOI: 10.2165/00003495200666080-00010.

3. Ferrari, M. D.; Goadsby, P. J.; Roon, K. I.; Lipton, R. B. Triptans (Serotonin, 5-HT1B/1D Agonists) in Migraine: Detailed Results and Methods of A Meta-Analysis of 53 Trials. Cephalalgia 2002, 22 (8), 633-658. DOI: 10.1046/j.1468-2982.2002.00404.x.

4. Ferrari, M. D.; Roon, K. I.; Lipton, R. B.; Goadsby, P. J. Oral triptans (serotonin 5-HT1B/1D agonists) in acute migraine treatment: a meta-analysis of 53 trials. Lancet 2001, 358 (9294), 1668-1675. DOI: 10.1016/ S0140-6736(01)06711-3.

5. Preetam, A.; Devi, A. S.; Sarbajna, R. M.; Suryanarayana, M. V.; Radhakrishna, S.; Rao, K. N.; Datta, D.; Lakshmi, P. V. A. Crystallographic Studies of Eletriptan Hydrobromide: $\alpha$-Form, $\beta$-Form and its Physicochemical Characterisation. Mol. Cryst. Liq. Cryst. 2013, 570 (1), 128-147. DOI: 10.1080/15421406.2012.721326.

6. Elqidra, R.; Ünlü, N.; Çapan, Y.; Sahin, G.; Dalkara, T.; Hincal, A. A. Effect of polymorphism on in vitro-in vivo properties of carbamazepine conventional tablets. J. Drug Delivery Sci. Technol. 2004, 14 (2), 147-153. DOI: 10.1016/S1773-2247(04)50027-8.

7. Liebenberg, W.; de Villiers, M. M.; Wurster, D. E.; Swanepoel, E.; Dekker, T. G.; Lötter, A. P. The Effect of Polymorphism on Powder Compaction and Dissolution Properties of Chemically Equivalent Oxytetracycline Hydrochloride Powders. Drug. Dev. Ind. Pharm. 1999, 25 (9), 1027-1033. DOI: 10.1081/ DDC-100102265.

8. Aguiar, A. J.; Zelmer, J. E. Dissolution behavior of polymorphs of chloramphenicol palmitate and mefenamic acid. J. Pharm. Sci. 1969, 58 (8), 983-987. DOI: $10.1002 / j p s .2600580817$.

9. Snider, D. A.; Addicks, W.; Owens, W. Polymorphism in generic drug product development. Adv. Drug. Deliv. Rev. 2004, 56 (3), 391-395. DOI: 10.1016/j. addr.2003.10.010.

10. Singhal, D.; Curatolo, W. Drug polymorphism and dosage form design: a practical perspective. Adv. Drug. Deliv. Rev. 2004, 56 (3), 335-347. DOI: 10.1016/j.addr.2003.10.008.

11. Dissolution Testing of Immediate Release Solid Oral Dosage Forms; Guidance for Industry; U.S. Department of Health and Human Services, Food and Drug Administration, Center for Drug Evaluation and Research (CDER), U.S. Government Printing Office: Washington, DC, 1997.

12. Maggio, R. M.; Castellano, P. M.; Kaufman, T. S. A new principal component analysis-based approach for testing "similarity" of drug dissolution profiles. Eur. J. Pharm. Sci. 2008, 34 (1), 66-77. DOI: 10.1016/j. ejps.2008.02.009.

13. Khan, F.; Li, M.; Schlindwein, W. Comparison of In Vitro Dissolution Tests for Commercially Available Aspirin Tablets. Dissolution Technol. 2013, 20 (1), 4458. DOI: 10.14227/DT200113P48.

14. O'Hara, T.; Dunne, A.; Butler, J.; Devane, J. A review of methods used to compare dissolution profile data. Pharm. Sci. Technol. Today 1998, 1 (5), 214-223. DOI: 10.1016/S1461-5347(98)00053-4.

15. Costa, P.; Sousa Lobo, J. M. Modeling and comparison of dissolution profiles. Eur. J. Pharm. Sci. 2001, 13 (2), 123-133. DOI: 10.1016/S0928-0987(01)00095-1.

16. Adams, E.; Coomans, D.; Smeyers-Verbeke, J.; Massart, D. L. Application of linear mixed effects models to the evaluation of dissolution profiles. Int. J. Pharm. 2001, 226 (1-2), 107-125. DOI: 10.1016/ S0378-5173(01)00775-X.

17. Khan, K. A. The concept of dissolution efficiency. J. Pharm. Pharmacol. 1975, 27 (1), 48-49. DOI: 10.1111/j.2042-7158.1975.tb09378.x.

18. Sathe, P. M.; Tsong, Y.; Shah, V. P. In-Vitro Dissolution Profile Comparison: Statistics and Analysis, Model Dependent Approach. Pharm. Res. 1996, 13 (12), 1799-1803. DOI: 10.1023/A:1016020822093.

19. Pedersen, P. V.; Myrick, J. W. Versatile kinetic approach to analysis of dissolution data. J. Pharm. Sci. 1978, 67 (10), 1450-1455. DOI: 10.1002/jps.2600671034.

20. Immediate Release Solid Oral Dosage Forms, Scale-Up and Postapproval Changes: Chemistry, Manufacturing, and Controls, In Vitro Dissolution Testing, and In Vivo Bioequivalence Documentation; Guidance for Industry; U.S. Department of Health and Human Services, Food and Drug Administration, Center for Drug Evaluation and Research (CDER), U.S. Government Printing Office: Washington, DC, 1995. 